\title{
Rietveld refinement guidelines
}

\author{
L. B. McCusker, ${ }^{a} *$ R. B. Von Dreele, ${ }^{b}$ D. E. Cox, ${ }^{c}$ D. Louër ${ }^{d}$ And P. ScArdi ${ }^{e}$
}

${ }^{a}$ Laboratorium für Kristallographie, ETH, Zürich, Switzerland, ${ }^{b}$ LANSCE, Los Alamos National Laboratory, Los Alamos, NM, USA, ${ }^{c}$ Physics Department, Brookhaven National Laboratory, Brookhaven, NY, USA, ${ }^{d}$ Laboratoire de Chimie du Solide et Inorganique Moléculaire (UMR 6511 CNRS), Université de Rennes I, Rennes, France, and ${ }^{e}$ Dipartimento di Ingegneria dei Materiali, Universitá di Trento, 38050 Mesiano (TN), Italy.

E-mail:lynne.mccusker@kristall.erdw.ethz.ch

(Received 23 February 1998; accepted 22 July 1998)

\begin{abstract}
A set of general guidelines for structure refinement using the Rietveld (whole-profile) method has been formulated by the International Union of Crystallography Commission on Powder Diffraction. The practical rather than the theoretical aspects of each step in a typical Rietveld refinement are discussed with a view to guiding newcomers in the field. The focus is on $\mathrm{X}$-ray powder diffraction data collected on a laboratory instrument, but features specific to data from neutron (both constant-wavelength and time-of-flight) and synchrotron radiation sources are also addressed. The topics covered include (i) data collection, (ii) background contribution, (iii) peak-shape function, (iv) refinement of profile parameters, (v) Fourier analysis with powder diffraction data, (vi) refinement of structural parameters, (vii) use of geometric restraints, (viii) calculation of e.s.d.'s, (ix) interpretation of $R$ values and (x) some common problems and possible solutions.
\end{abstract}

\section{Introduction}

The results of two Rietveld refinement (Rietveld, 1969) round robins organized by the Commission on Powder Diffraction (CPD) of the International Union of Crystallography were published in 1992 by Hill and in 1994 by Hill \& Cranswick. These studies were designed to evaluate a cross section of the currently used Rietveld software, to examine the effect of different refinement strategies, to assess the accuracy and precision of the parameters obtained in a Rietveld refinement, and to compare different instruments and methods of data collection. The results highlighted some of the problem areas and led to a series of recommendations regarding both data-collection and refinement strategies.

The CPD has now formulated a set of general guidelines that encompasses the initial recommendations regarding Rietveld refinement and supplements them with some explanatory and/or cautionary notes. These guidelines cover the practical aspects of the Rietveld method and focus on data collected on a laboratory instrument. With the advent of graded multilayer optics and linear position-sensitive detectors, capillary measurements in the laboratory are being used with increasing frequency, so both reflection (BraggBrentano) and transmission (Debye-Scherrer, Guinier) geometries are considered. Features that are specific to data from neutron (constant-wavelength and/or time-offlight) and synchrotron (or parallel-beam laboratory) sources are addressed separately where appropriate. While the use of Rietveld refinement for quantitative analysis is not dealt with specifically, the guidelines are also valid for this application. However, for additional information on this topic, the reader is referred to the paper by Hill (1991).

In the following sections, the practical aspects of each step of a typical Rietveld refinement (including some of the critical factors in the data collection itself) are discussed, in the hope that the rapidly growing powder diffraction community can benefit from a relatively concise presentation of the pitfalls awaiting them and of some of the possible solutions. Each topic is handled separately to enable easy reference. A newcomer to Rietveld refinement is encouraged to start with the refinement of the structure of a standard material such as $\mathrm{ZrO}_{2}$ (Hill \& Cranswick, 1994) and to compare the results with the published ones. For more detailed information, the reader is referred to The Rietveld Method edited by R. A. Young (1993) and the monograph Modern Powder Diffraction edited by Bish \& Post (1989). Guidelines for the publication of the results of Rietveld analyses can be found in the paper by Young et al. (1982).

It has been assumed that the Rietveld refinement software used is reliable. For an up-to-date list of Rietveld refinement programs, see the CPD WWW pages (current location http://www.iucr.org/iucr-top/comm/ $\mathrm{cpd} /$ ). Should the WWW site change, the link from the IUCr site (http://www.iucr.org/) will be modified accordingly.

\section{Data collection}

For a Rietveld refinement, it is essential that the powder diffraction data be collected appropriately. Factors to 
consider prior to data collection are the geometry of the diffractometer, the quality of the instrument alignment and calibration, the most suitable radiation (e.g. conventional X-ray, synchrotron X-ray or neutron), the wavelength, appropriate sample preparation and thickness, slit sizes, and necessary counting time. If the relative intensities and/or the $2 \theta$ values ( $d$ spacings) are not correct, no amount of time spent on the structure refinement will yield sensible results. It is not the purpose of this paper to delve into the intricacies of data collection, but it is perhaps appropriate to indicate briefly where common errors occur.

For Bragg-Brentano geometries, it is important that the incident beam be kept on the sample at all angles to ensure a constant-volume condition. Quite often, the divergence slits used are too wide and the beam hits the sample holder at low angles, so the intensities measured at these angles are too low. Some programs can correct for this, but most do not. If a correction is used, the shape of the sample holder (i.e. circular or rectangular) must also be taken into account. It should not be assumed that using a rotating circular sample will eliminate the problem. For efficient data collection, smaller slits can be used at low angles and wider ones at higher angles, provided that the different ranges are appropriately scaled to one another and the e.s.d.'s are correctly calculated. Alternatively, each section can be treated as a separate data-set in a multi-data-set Rietveld refinement. The latter approach also eliminates any problems caused by different resolution characteristics in the different sections.

Many laboratory diffractometers are equipped with automatic divergence slits, which open as a function of $2 \theta$. While there is a substantial gain in diffracted intensity at high angles with such slits, there is also a progressive deterioration of the parafocusing condition and therefore of the resolution as $2 \theta$ increases. In Bragg-Brentano geometry, the flat specimen surface is tangent to the focusing circle and, as $2 \theta$ increases, the radius of this circle diminishes. At the same time, the illuminated area of the sample is reduced, so the flatsample effect on data resolution is minor, whatever the diffraction angle. However, if varying slit widths are used, a progressive angular-dependent defocusing occurs and the quality of the data deteriorates. Furthermore, if the measured intensities are to be used in a Rietveld refinement, the slit opening needs to have a precision of at least $1 \%$ or, in other words, be reproducible to a few microns over the entire $2 \theta$ range. For these reasons, the use of such slits for a Rietveld refinement is not recommended.

To ensure good counting statistics throughout an $\mathrm{X}$ ray powder diffraction pattern, more time should be spent on data collection at high angles where the intensities are lower. An appropriate data-collection strategy will depend on the nature of the sample (e.g. how well it scatters, how quickly the pattern degrades, peak-broadening effects and the degree of peak overlap). There should be at least five steps (but generally not more than ten) across the top of each peak (i.e. step size $=\mathrm{FWHM} / 5$, where FWHM is the full width at half-maximum), the time per step should approximately compensate for the gradual decline in intensity with $2 \theta$ (Madsen \& Hill, 1994; Shankland et al., 1997) and the maximum $2 \theta$ value should be chosen to give the maximum useful data (i.e. as high as possible). Care must be taken that the e.s.d.'s are properly calculated for different counting times.

Another problem is that of sample transparency. The assumption for reflection geometry is that the sample is 'infinitely thick' (i.e. the X-ray beam is totally absorbed by the sample). If the sample contains only light elements, this condition might not be fulfilled. Then the constant-volume assumption is not valid and the intensities measured at high angles will be too low unless an appropriate correction is introduced at the refinement stage. For such materials, transmission geometry is usually to be preferred.

On the other hand, heavily absorbing samples can be a problem in a transmission set-up, because the incident beam cannot penetrate the whole sample. In this case, the sample may have to be diluted with a light-element material (e.g. diamond powder or glass beads). For such samples, reflection geometry is usually to be preferred. However, for highly absorbing materials, a potential source of error in reflection geometry is that of surface roughness. This can reduce the intensity of low-angle reflections and lead to anomalously low thermal parameters in refinement. Corrections have been suggested by Suortti (1972), Masciocchi et al. (1991) and Pitschke et al. (1993), and have been implemented in some programs.

Preferred-orientation effects can be difficult to eliminate, especially for flat-plate specimens (even when back or side loaded), and these can also lead to incorrect intensity measurements. If the intensities show a strong $h k l$ dependence (e.g. all $00 l$ reflections are strong and all $h k 0$ weak), preferred orientation of the crystallites should be suspected. Altomare et al. (1996) and Peschar et al. (1995) have developed methods for analyzing powder diffraction data for preferred-orientation effects. Although many Rietveld refinement programs allow refinement of a preferred-orientation parameter with respect to a specific crystallographic vector based on the March model (Dollase, 1986), this is usually only a crude approximation to reality, so elimination (or minimization) of the problem experimentally is to be preferred. More sophisticated corrections using spherical harmonics (Järvinen, 1993; Von Dreele, 1997) have been introduced in some programs. Rotation of a horizontally mounted capillary (usual set-up at a synchrotron) can virtually eliminate preferred-orientation effects, although long needles may still tend to remain aligned along the capillary axis. If other considerations 
dictate that a flat-plate sample be used, spray drying the sample to minimize preferred orientation should be considered. Rotation of a flat-plate sample in its own plane will not correct preferred-orientation effects.

An ideal particle size within a powder sample is circa 1-5 $\mu \mathrm{m}$. If large crystallites are used, there are fewer crystallites in the sample and nonrandomness (i.e. not all crystallite orientations are equally represented) may become a problem. This can cause nonsystematic inaccuracies in the relative intensities that, unlike preferredorientation effects, cannot be corrected at the refinement stage. Sample rotation will improve the particle statistics and is strongly recommended. If smaller particles are used, line-broadening effects due to crystallite size begin to become apparent. If there are one or more large crystallites within a sample of smaller ones ('rocks in the dust'), the relative intensities of the reflections originating from the larger crystallites will be too high in comparison with the other reflections and as with nonrandomness, no correction can be applied at the refinement stage. For more information on sample preparation, see Bish \& Reynolds (1989) or Buhrke et al. (1998).

The $2 \theta$ values should be carefully calibrated using several peaks from a standard material (e.g. NIST Si standard SRM 640b and/or fluorophlogopite mica SRM 675 for low angles). Almost any diffractometer can be adjusted so that the deviations of the measured peak positions from the correct ones are less than $0.01^{\circ}(2 \theta)$. Furthermore, the diffractometer should be set-up to give a low background and maximum peak resolution (small peak widths) if a complex pattern with significant reflection overlap is to be measured [e.g. small receiving slits, pre-detector Soller slits if available, receiving slit on the focusing circle, $0.3 \mathrm{~mm}$ capillary or smaller for transmission geometry, monochromatic radiation (e.g. $\mathrm{Cu} K \alpha_{1}$ rather than $\mathrm{Cu} K \alpha_{1,2}$, if possible)]. Although longer data-acquisition times are required with monochromatic radiation, its use is particularly advantageous: the number of lines in the pattern is halved (so the severe overlap of reflections begins at higher angles) and the background is lower. If both $\alpha_{1}$ and $\alpha_{2}$ components are used in the data collection, the spectral distribution (intensity ratios) and dispersion (profile changes as a function of $2 \theta$ ) must also be calibrated.

Any temptation to smooth the diffraction data before doing a Rietveld refinement must be resisted. Smoothing introduces point-to-point correlations (offdiagonal weight matrix elements) which will give falsely lowered e.s.d.'s in the refinement. For more information on data-collection aspects, the reader is referred to the results of the second round robin (Hill \& Cranswick, 1994), to the review on powder diffraction by Langford \& Louër (1996) and to the monograph edited by Bish \& Post (1989) mentioned earlier. For information regarding the effect of systematic errors on powder diffraction data see Wilson (1963).
Synchrotron. There are a number of options for data collection at a synchrotron facility, which may not be familiar to users of conventional laboratory equipment and which should be discussed in detail with the beamline scientist prior to starting an experiment. These options involve a compromise between resolution, intensity and peak-to-background discrimination, and among the many factors to be considered are (i) the diffraction geometry (e.g. monochromator, crystal analyzer, single slits, Soller slits), (ii) detectors [e.g. scintillator, semiconductor, linear or curved positionsensitive detector (PSD), imaging plate (IP), chargecoupled detector (CCD)], (iii) sample geometry (e.g. flat-plate in symmetric or grazing incidence reflection, flat-plate in symmetric or normal transmission, capillary) and (iv) wavelength (typically $0.3-2.5 \AA$ for a bending-magnet beamline, depending on the energy of the synchrotron source).

The best instrumental resolution [circa 0.01$\left.0.02^{\circ}(2 \theta)\right]$ and peak-to-background discrimination are obtained with a crystal analyzer (CA) mounted in the diffraction path between the sample and the detector, but only at the expense of considerably reduced counting statistics. However, the relatively low counting rates in this case can be compensated for if a multianalyzer detector (Toraya et al., 1996; Hodeau et al., 1996) is available. The best counting statistics are obtained with area detectors such as an IP, a PSD or a CCD.

The wavelength and zero offset should be calibrated with a reference material. The Si SRM 640b standard gives significantly broadened peaks, whereas the NIST $\mathrm{LaB}_{6}$ standard SRM 660 gives close to instrumental resolution and is probably a better choice. Commonly used wavelengths at second-generation sources range between 0.7 and $1.2 \AA(1.54 \AA$ is unlikely to be an optimal choice because of excessive attenuation). At a third-generation or high-energy source, even shorter wavelengths down to circa $0.3 \AA$ are available.

Problems with highly crystalline samples, in which there is insufficient sampling of grains suitably oriented for diffraction, may be exacerbated at a synchrotron source because of the highly collimated nature of the incident monochromatic beam; it is thus essential to rotate or rock the sample during data collection. For capillary samples, the diameter of the capillary and the wavelength should be chosen so that $\mu R$ (where $\mu$ is the linear absorption coefficient and $R$ is the diameter of the cylinder) is not too large (see International Tables for Crystallography, 1995; Sabine et al., 1998). If $\mu R$ is larger than 1 , the simple form of the absorption correction is no longer valid and a more sophisticated correction is needed. Corrections of this type are not currently implemented in most Rietveld programs, so they need to be applied to the raw data prior to refinement. In practice, it is difficult to load capillaries less than $0.2 \mathrm{~mm}$ in diameter and thus short wavelengths (or samples 
diluted with diamond powder or glass beads) are required for samples containing heavier elements. The proximity of absorption edges must be kept in mind. Typical packing fractions of capillary samples range between 20 and $50 \%$, and it is strongly recommended that the approximate density be determined directly from the measured sample dimensions and weight so that quantitative absorption corrections can be made in the subsequent data analysis. The feasibility of using capillary samples is often a great advantage in synchrotron X-ray experiments and data collected in this way (especially at shorter wavelengths) generally seem to give the most satisfactory refinements.

Anomalous (resonant) scattering techniques can be used to enhance the contrast between elements close to one another in the Periodic Table and thereby serve as a probe of cation distribution (or even oxidation states in some cases). If the $K$ or $L_{\text {III }}$ edge is accessible at the beamline, it is recommended that for such experiments at least two data-sets be collected: one about 10-20 eV below the absorption edge (where the scattering factor may be reduced by 5-20 electrons depending on the atomic number) and one at least $1 \mathrm{keV}$ below the edge, or, if feasible, well above the edge.

Because a synchrotron beam decays with time, the incident-beam intensity must be monitored during an experiment and the resulting data normalized for this decay. Data can be collected with constant monitor counts (variable time) or constant time (variable monitor counts) per step. In the latter case, the measured intensity must be normalized using the monitor counts and the e.s.d.'s calculated accordingly. A word of warning: not all programs can accommodate the input of e.s.d.'s. If this is the case, constant monitor counts should be used to collect the data. Otherwise, the weighting scheme used in refinement will be incorrect. The value of the polarization factor of the incident beam is another quantity which will be needed in the subsequent refinement stage and the beamline scientist should be consulted as to the appropriate value to use.

Constant-wavelength neutron. The most common diffractometer geometry used for collection of constantwavelength neutron powder diffraction data is the Debye-Scherrer arrangement, where one detector, or more commonly an array of detectors, is moved about a cylindrical sample. The neutron wavelength is selected by an appropriate monochromator and the instrumental diffraction peak width is controlled by a choice of Soller collimators located between the neutron source and the monochromator and between the sample and the detector. Certain choices of monochromator crystals can lead to the contamination of the diffraction pattern by $\lambda / 2$ or $\lambda / 3$ radiation; filters are usually used to eliminate this higher-order effect.

Generally, the resolution is poorer than that routinely obtained with X-rays; thus sample-broadening effects have less of an impact on the quality of a Rietveld refinement. For most elements in the Periodic Table, neutron absorption coefficients are roughly four orders of magnitude lower than the corresponding X-ray absorption coefficients. Consequently, sample sizes for neutron powder diffraction can be much larger than those used for X-ray Debye-Scherrer experiments. Typical samples range from 5 to $20 \mathrm{~mm}$ in diameter and from 5 to $50 \mathrm{~mm}$ in length, depending on the overall size of the incident neutron beam as well as the amount of sample available. Because neutron sources are very much weaker than X-ray sources, the large sample size is essential. The combination of large sample volume and high neutron penetration reduces the problem of preferred orientation considerably, but it cannot always be completely discounted as a possible systematic effect on the diffracted intensities. Similarly, crystallite-size effects on counting statistics are much less significant because the large sample volume and large incidentbeam divergence give a good powder average, so rotation of the sample during data collection is not necessary.

Many neutron powder diffraction experiments are performed in special environments (e.g. temperature, pressure, surrounding gas, etc.). Particular attention must be paid to the choice of materials used to build such environmental chambers so that interference from additional diffraction patterns from the construction materials is kept to a minimum. The fact that many metals commonly used for cryostats, for example, have been rolled into sheet form is particularly troublesome. The diffraction patterns from such materials will display strong texture effects, which are difficult to treat properly in a Rietveld refinement.

Because neutrons are scattered by the atomic nuclei, the only fall-off in intensity as a function of scattering angle is due to the thermal motion of the atoms. Consequently, the scattered intensity at high angles is only slightly lower than that at low angles and thus there is little to be gained by variable-speed data collections as suggested above for X-ray diffraction experiments. However, the step size should be chosen as noted above with five or more steps across the FWHM.

Calibration is essential to establish the neutron wavelength. The Si SRM 640b standard and several other materials are suitable for both wavelength and instrumental broadening calibration. However, the $\mathrm{LaB}_{6}$ standard should not be used, because boron is a strong neutron absorber. For instruments with an array of detectors, a calibration to establish the angular offset and counting efficiency of each detector in the array is also required. Each diffraction pattern obtained from the single detectors in an array can also be treated individually in a multipattern Rietveld refinement.

Time-of-flight neutron. Neutron powder diffractometers using the time-of-flight (TOF) principle operate in a very different mode than do conventional constantwavelength X-ray or neutron diffractometers. The 
detectors are located at fixed scattering angles and the diffraction pattern is recorded as a function of the time delay from the start of a neutron burst at the source. The repetition frequency of the source is generally fixed by the operation of the neutron facility itself. This, in combination with the neutron flight path, will dictate the range of neutron wavelengths seen by the diffractometer, which in turn dictates the range of $d$ spacings accessible by a particular detector. The incident neutron spectrum is a strong function of the wavelength and can be four orders of magnitude larger at short wavelengths (i.e. $1 \AA$ ) compared with long wavelengths (e.g. $8 \AA$ ). The instrument layout is similar to a Debye-Scherrer camera with detectors commonly positioned at a wide variety of scattering angles on both sides of the incident beam. The resolution characteristics of these instruments are very different from those of conventional ones, in that an individual detector will give diffraction peak widths that are essentially proportional to the reflection $d$ spacing; this resolution will vary from detector to detector as a function of the scattering angle.

The diffraction profiles are strongly asymmetric because of the time structure of the neutron pulse from the source. Consequently, calibration procedures are more complex. The shape of the incident spectrum is established by measuring the pattern obtained from a 'null' scatterer (usually vanadium). This measurement determines the product of the wavelength response of the detectors and the incident neutron wavelength spectrum. The relationship between neutron time-offlight and the $d$ spacing of the observed Bragg peaks is established using standards such as Si SRM 640b. This relationship depends on both scattering angle and the length of the neutron flight path; thus it is very sensitive to the sample location within the diffractometer. Sample-position errors or even the displacement of the effective scattering centre can introduce a bias in the values of the lattice parameters obtained from a Rietveld refinement (or any other peak-fitting procedure) that is much larger than the precision. Only by using internal standards can this problem be overcome. Some refinement techniques to reduce this bias to a minimum are discussed in the profile parameter section.

\section{Background contribution}

There are basically two approaches to dealing with the background in a powder diffraction pattern. It can either be estimated by linear interpolation between selected points between peaks and then subtracted, or it can be modelled by an empirical or semiempirical function containing several refinable parameters. Both have their advantages and disadvantages. For a simple pattern where most peaks are resolved to the baseline, both methods tend to work well and the fit is easily verified with a plot.
For complex patterns with a high degree of reflection overlap, however, the majority of the peaks are not resolved to the baseline, so the estimation of the background is difficult. This means that if a backgroundsubtraction approach is used, the background usually has to be re-estimated and re-subtracted several times during a refinement. The background variance is also difficult to estimate and is often assumed to be zero. However, it should be taken into account if the weighting scheme used in the refinement [usually $1 / \sigma^{2}(I)$ ] is to be correct. While background subtraction can be a somewhat cumbersome approach to background correction and may seem to be rather unsophisticated, it has the advantage of being flexible.

It might appear that refining the background would be the preferred method, because this would eliminate some of the problems inherent to background subtraction (i.e. the background and structural parameters can be refined simultaneously and the standard deviations estimated in the usual way). However, the polynomial functions usually used for this purpose are largely or entirely empirical. If the polynomial happens to describe the background well, then, as might be expected, this procedure also works well; but if it does not, no amount of refining the coefficients of the polynomial (or increasing the order of the polynomial) can correct the problem and the refinement will not proceed satisfactorily. In such a case, background subtraction is the better approach. Of course, it is also possible to use the two techniques in combination. A simple empirical function can be used to fit the background and then the deviations described by a sequence of fixed points. The advantage is that the magnitude of the fixed part is minimized and thus the impact of its inherent arbitrariness on the refinement is reduced.

Synchrotron. Because of the excellent signal-to-noise discrimination that is routinely obtained with synchrotron radiation (especially with $\mathrm{CA}$ geometry), the background in a synchrotron experiment tends to be more sensitive to structural disorder and fluctuations than that in a laboratory X-ray experiment. Background interpolation and subtraction usually yields better results than refinement with arbitrary polynomial functions. The high resolution allows the background intensities between peaks to be estimated more reliably.

Constant-wavelength neutron. The prime contributor to the background in constant-wavelength neutron experiments is incoherent scattering from the sample container and from the sample itself. The sample containers are generally made of vanadium (preferably $<0.1 \mathrm{~mm}$ thick) because this element has essentially no coherent scattering (no Bragg peaks), but it does have appreciable incoherent scattering which contributes to the background. Aluminium or fused silica cans are also used sometimes, but the former gives additional Bragg peaks and the latter contributes a nonuniform feature to the background. Incoherent scattering from the sample 
is particularly evident in materials containing $\mathrm{H}$, which have not been deuterated. Deuteration is essential to reduce the background and it is important to know the degree of deuteration because of the effect of the H/D ratio on the scattering length $(\mathrm{H}$ has a positive and $\mathrm{D}$ a negative scattering length; at circa $2 \mathrm{H} / 1 \mathrm{D}$ the effective scattering length is zero).

TOF neutron. For a neutron TOF experiment, the appearance of the diffraction pattern (including background) will depend on how the diffraction data are presented. The 'raw' counts will generally be strongest at the short TOF end of the pattern (lowest $d$ spacing), because the incident spectrum is strongest at short TOF. The pattern will tail off rapidly at large TOF (large $d$ spacing), because of the fall-off in incident intensity. This gives the appearance of a high background and high Bragg intensity at short TOF. Consequently, one useful way of presenting neutron TOF data for publication is to give the intensity modified by subtracting the fitted background from both the observed intensity and the best Rietveld refinement fit and plotting the result. On the other hand, if the intensity is normalized using the incident intensity spectrum, then the background will be revealed as being nearly flat, and the Bragg peaks at longest TOF (largest $d$ spacing) will usually be the largest ones in the pattern with the peak intensities tailing-off very sharply at small TOF (low $d$ spacing). This effect arises from the form of the Lorentz correction for TOF data, where

$$
L=d^{4} \sin \theta,
$$

which strongly enhances the scattered intensity for large- $d$-spacing reflections.

\section{Peak-shape function}

Of course, an accurate description of the shapes of the peaks in a powder pattern is critical to the success of a Rietveld refinement. If the peaks are poorly described, the refinement will not be satisfactory. The peak shapes observed are a function of both the sample (e.g. domain size, stress/strain, defects) and the instrument (e.g. radiation source, geometry, slit sizes), and they vary as a function of $2 \theta$. In certain cases, they can also vary as a function of $h k l$ indices. Accommodating all of these aspects in a single peak-shape description is nontrivial and compromises are often made.

Of the analytical peak-shape functions investigated, the pseudo-Voigt approximation of the Voigt function is probably the most widely used for X-ray and constantwavelength neutron data (Thompson et al., 1987). The pseudo-Voigt function is simply a linear combination of Lorentzian and Gaussian components in the ratio $\eta /(1-\eta)$, where $\eta$ is the pseudo-Voigt mixing parameter. This appears to describe the symmetrical part of an $\mathrm{X}$-ray diffraction peak quite well. However, an additional function to allow a more precise description of asymmetry due to axial divergence of the diffracted beam at low angles is essential (Finger et al., 1994, and references therein). For X-ray diffraction lines dominated by instrumental broadening, $\eta$ usually varies in a linear manner, from a dominant Gaussian component at low angles to a Lorentzian trend at high angles. If a Pearson VII peak-shape function is used, the exponent $m$ varies differently, but the same trends in line shape are observed (Louër \& Langford, 1988). An advantage of the pseudo-Voigt or Voigt functions is that they allow the Gaussian and Lorentzian components of the peak shape to be introduced in a physically intuitive way to model the overall line broadening, which furnishes useful information about the microstructure with respect to size and strain effects. The Pearson VII function does not have this physical basis. However, both can be used to describe the peak shape for Rietveld refinement.

A pragmatic alternative to an analytical peak-shape function is an experimental or 'learned' one (Hepp \& Baerlocher, 1988). In this case, a single peak (one reflection) is selected from the diffraction pattern as a standard peak. This peak is fitted according to some criteria (e.g. two inflection points, one maximum etc.), the calculated curve is divided into a symmetric and an asymmetric part, and these values are stored in tabulated form. Such a standard peak automatically reflects contributions from both the sample and the instrument, because it has been taken from the experimental data. This means, of course, that the individual contributions from the sample and the instrument cannot be separated as they can with a pseudo-Voigt function. The standard peak is then used to describe all of the peaks in the pattern by varying the FWHM and/or the ratio of the symmetric to the asymmetric part as a function of $2 \theta$ (and possibly of $h k l$ ).

Regardless of the type of peak-shape function selected, the range of the peak (i.e. when it no longer contributes significant intensity to the diffraction pattern) must be established. As a rule of thumb, a peak can be considered to be down to background level when the intensity is less than $0.1-1.0 \%$ of the peak maximum. The appropriate percentage depends upon the peak shape. If the 'tails' of the peaks are long (i.e. high Lorentzian character), a wider range will be required than for peaks with more Gaussian character. To illustrate the difference, the percentages of the total area of a purely Lorentzian and of a purely Gaussian peak are given for different ranges in Table 1 . The range needed depends upon the FWHM of the peak; thus it is usually expressed as an integral number of FWHM values, though some programs use a fraction of the peak height. Typically, the value will range from 10 to $20 \times$ FWHM depending upon the Lorentzian character of the peak. Thus, a reflection with an FWHM of $0.10^{\circ}(2 \theta)$ contributes to the diffracted intensity over a range of at least $1.0-2.0^{\circ}(2 \theta)$ (and sometimes much more). This param- 
Table. Effect of peak range on integrated intensity for pure Lorentzian and Gaussian peak shapes

\begin{tabular}{lcc}
\multicolumn{3}{c}{ Pure Lorentzian peak shape } \\
\hline \multicolumn{2}{c}{ Peak range } \\
\% Peak height & No. FWHM & \% Total area \\
0.990 & 5 & 93.67 \\
0.249 & 10 & 96.84 \\
0.111 & 15 & 97.90 \\
0.062 & 20 & 98.43 \\
0.040 & 25 & 98.75 \\
0.028 & 30 & 98.96 \\
0.020 & 35 & 99.11 \\
0.015 & 40 & 99.22
\end{tabular}

\begin{tabular}{lll}
\multicolumn{3}{c}{ Pure Gaussian peak shape } \\
\hline \multicolumn{2}{c}{ Peak range } \\
\% Peak height & No. FWHM & \% Total area \\
10.57 & 1.8 & 96.60 \\
6.17 & 2.0 & 98.17 \\
3.40 & 2.2 & 99.07 \\
1.82 & 2.4 & 99.53 \\
0.90 & 2.6 & 99.79 \\
0.43 & 2.8 & 99.90 \\
0.19 & 3.0 & 99.96 \\
0.079 & 3.2 & 99.98
\end{tabular}

eter is often set at much too low a value, resulting in a severe underestimation of the reflection intensity. If the 'tails' of high-intensity reflections are cut off prematurely in the profile plot (see Fig. 1), this is a good indication that the range has been set at too small a value.

Synchrotron. In general, the symmetric part of the peak-shape function is very well accounted for by the pseudo-Voigt function described above (the full Voigt convolution may give an even better fit). For very high resolution data such as those obtained with a crystal analyzer, the instrumental contribution to the peak shape is minimal and the peaks tend to be highly Lorentzian (i.e. dominated by sample effects). For these data, an initial peak range of at least 40 FWHM is recommended. Because the intrinsic peak widths are extremely narrow, asymmetric broadening at low angles may become very pronounced and must be corrected for properly.

TOF neutron. The peak-shape functions employed for Rietveld refinement with neutron TOF data are more complex than those used for constant-wavelength neutron or X-ray data, because the neutron-pulse structure imposes an additional and asymmetric broadening effect. A simple empirical description, in which a back-to-back paired set of exponentials is convoluted with a Gaussian, has been developed by Von Dreele et al. (1982),

$$
H(\Delta T)=N[\exp (u) \operatorname{erfc}(x)+\exp (v) \operatorname{erfc}(y)]
$$

where $H$ is the peak-shape function for a single reflection, erfc is the complementary error function, and the parameters $N, u, v, x$ and $y$ have various functional dependencies on the $d$ spacing of the reflection, the scattering angle of the detector and various characteristics of the neutron source, as well as the offset of the profile point from the reflection position, $\Delta T$.

\section{Profile parameters}

If only a partial structural model is available, the calculated intensities may deviate significantly from the observed ones, and this can complicate the initial refinement of profile parameters (changes in FWHM and peak asymmetry as a function of $2 \theta, 2 \theta$ correction,

unit-cell parameters). In this case, it is probably best to use a structure-free approach, in which the intensities of the reflections are simply adjusted to fit the observed ones (Le Bail et al., 1988), to obtain initial values for the profile parameters. This option (usually referred to as a Le Bail refinement), which can also be used to extract a list of integrated intensities from a powder pattern for structure determination, has now been included in most modern Rietveld programs. Sivia \& David (1994) have developed an alternative to the Le Bail algorithm using least-squares refinement (Pawley, 1981) coupled with Bayesian arguments. This method enables the e.s.d.'s of the reflection intensities to be estimated more correctly and calculates the covariances between overlapping reflections. For structure determination this additional information is particularly useful. Whichever algorithm is used, initial values for the profile parameters can be determined. These parameters can then be further refined at a later stage when a reliable structural model has been established. If the structural model is complete and the relative intensities match the observed ones well enough, the initial profile-parameter refinement can also be performed using the structural model. In this case, the calculated pattern must first be scaled to the observed one.

Before the refinement of structural or profile parameters can be started, the positions of the observed and calculated peaks must match well. That is, the unit-cell parameters and the $2 \theta$ correction (which includes both the zero offset and the deviations in $2 \theta$ caused by displacement of the sample from the centre of the $2 \theta$ circle) need to be refined first. These parameters can be highly correlated; thus it is sometimes necessary to determine one or the other independently. This is probably best done by mixing a standard material (e.g. NIST Si standard SRM 640b) with the sample and collecting a second data-set. In this way, the $2 \theta$ correction for the second measurement can be established from the Si peaks and the cell parameters of the material of interest refined. These refined cell parameters can then be used with confidence in the structure refinement using the data collected on the pure sample and any misfit in $2 \theta$ can be adjusted by refining the $2 \theta$ correction parameters. If an internal standard cannot be used for some reason (e.g. insufficient sample, sensitive sample, 
etc.), at least the $2 \theta$ calibration of the instrument should be checked carefully using an external standard. If the initial cell-parameter refinement should prove to be difficult, a Le Bail (structure-free) refinement using only the low-angle data may help. For complex structures involving a high degree of reflection overlap, artificially sharpening the calculated pattern (e.g. setting FWHM values to irca $80 \%$ of their true values) will sometimes help the refinement by forcing a good fit of the maxima. However, this will only work if the peak positions are already approximately correct. It should be emphasized that unless the peak positions of the observed and calculated patterns match fairly well, Rietveld refinement cannot and will not work.

The appropriate function for the correction of $2 \theta$ due to sample displacement depends upon the geometry of the instrument. For example, for Bragg-Brentano geometry with a flat-plate sample it is

$$
\Delta 2 \theta=(-2 s \cos \theta) / R
$$

where $s$ is the displacement of the specimen and $R$ is the radius of the goniometer circle, and for Debye-Scherrer geometry with a capillary sample it is

$$
\Delta 2 \theta=(x \sin 2 \theta-y \cos 2 \theta) / R
$$

where $x$ and $y$ are the respective displacements of the capillary from the centre of the $2 \theta$ circle.

Once a good match of the peak positions has been achieved, the other profile parameters (first peak width and then peak asymmetry as a function of $2 \theta$ ) can be refined. The variation of the FWHM of the Gaussian component of the peak shape as a function of $2 \theta$ is usually modelled with the equation derived by Caglioti et al. (1958) for low-resolution neutron data,

$$
\mathrm{FWHM}^{2}=U \tan ^{2} \theta+V \tan \theta+W,
$$

and that of the Lorentzian part by

$$
\mathrm{FWHM}=X \tan \theta+Y / \cos \theta .
$$

While these functions are simple and usually work well, they cannot be used to model anisotropic line broadening (i.e. $h k l$-dependent line broadening) and may not describe the $2 \theta$ dependence very well. In such cases, a more flexible approach (e.g. input and/or refinement of effective diffracting domain shape and anisotropic strain) is necessary. For further discussion of this topic, the reader is referred to Delhez et al. (1993), RodriguezCarvajal (1996), Langford \& Louër (1996), Le Bail (1998), Scardi (1998) and Stephens (1999).

Incorrect profile parameters yield characteristic difference profiles; thus the examination of a suitably enlarged profile plot can be very useful in determining which parameters need further refinement. In Figs. 2-6, various misfits of a calculated profile to the observed data are illustrated for the same (somewhat asymmetric) peak. A good fit is shown in Fig. 2(a) and the effect of a pure intensity difference in Figs. 2(b) and 2(c); the effect of incorrect peak widths is shown in Fig. 3; the effect of too little peak asymmetry in Fig. 4; and the effect of a $2 \theta$ mismatch in Fig. 5. In a real refinement, the misfits are rarely caused by a single variable and so two examples of combinations of incorrect profile parameters are given in Fig. 6.

Synchrotron. Refinement of the lattice parameters and zero offset generally presents no problems, especially when sample-displacement errors are eliminated by the use of CA or Soller-slit geometry. When area detectors are used, very specialized techniques of data processing are required to produce a standard powder profile and there may be additional sources of error that are beyond the scope of this discussion. Because of the predominantly Lorentzian character of high-resolution data, refinement of the Lorentzian half-width parameters $X$ and $Y$ is usually straightforward, but unconstrained refinement of the Gaussian parameters $U, V$ and $W$ may lead to nonphysical results, or at worst, complete failure of the refinement. In such cases, some kind of constraint function should be applied, or the

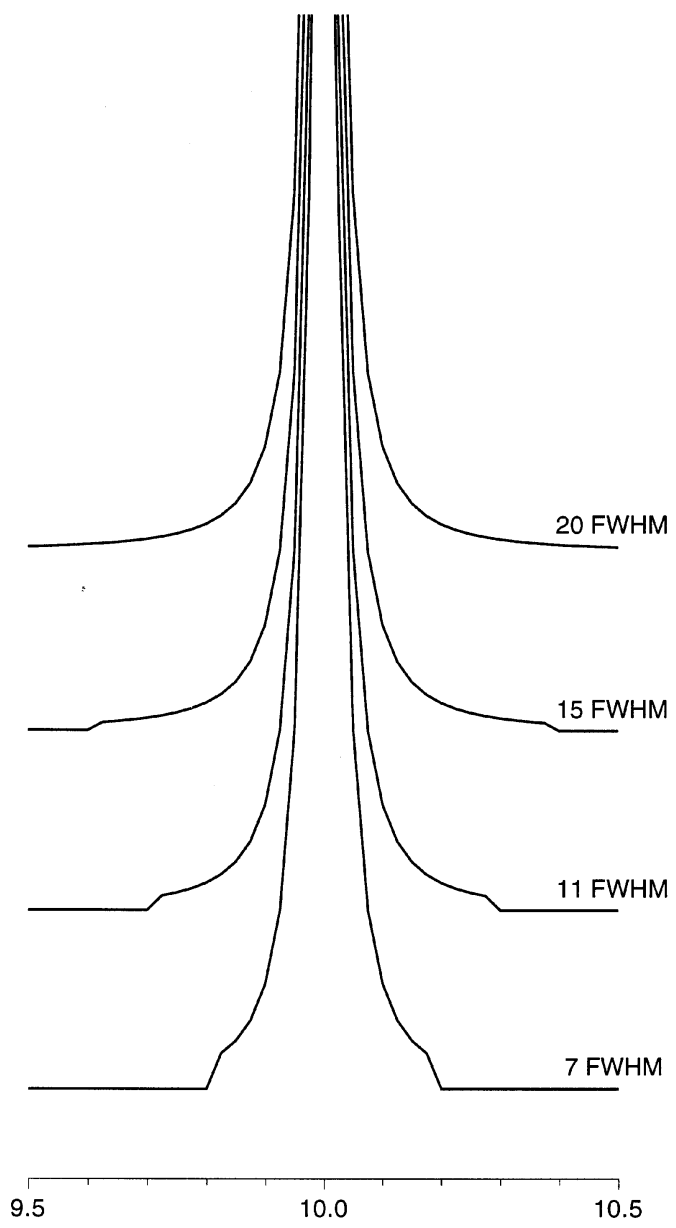

Fig. 1. A Lorentzian peak calculated using a series of different peak ranges. The truncation effect with decreasing numbers of FWHM is readily apparent. 
parameters fixed at the instrumental values. Because of the very narrow peak widths, anisotropic line broadening is much more likely to be visible in a synchrotron experiment and careful attention must be given to the choice of a suitable model.

Constant-wavelength neutron. The peak-shape functions used for constant-wavelength neutron diffraction data are essentially the same as those described for Xrays. However, $U, V$ and $W$ make a far more dramatic contribution to the profile because constant-wavelength

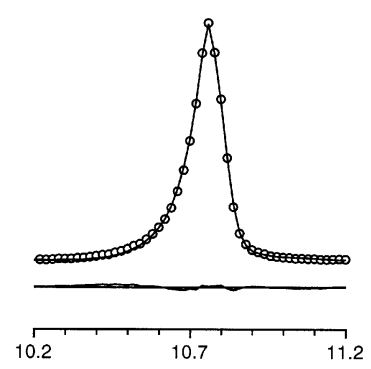

(a)

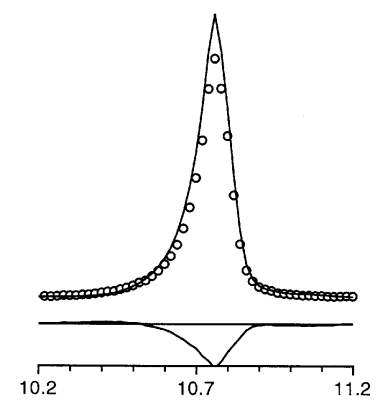

(b)

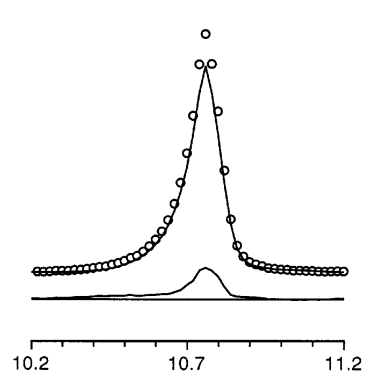

(c)
Fig. 2. The observed (circles), calculated (line) and difference (bottom) profiles for $(a)$ a good fit of a peak, $(b)$ a calculated intensity that is too high and $(c)$ a calculated intensity that is too low. The characteristic difference profile for an intensity is either positive or negative and concentrated at the centre of the peak.

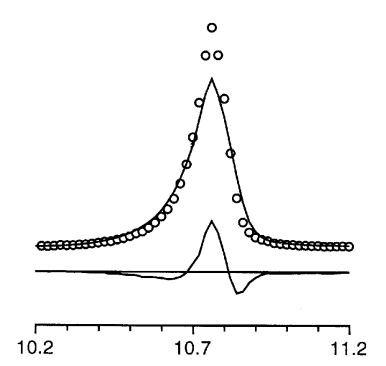

(a)

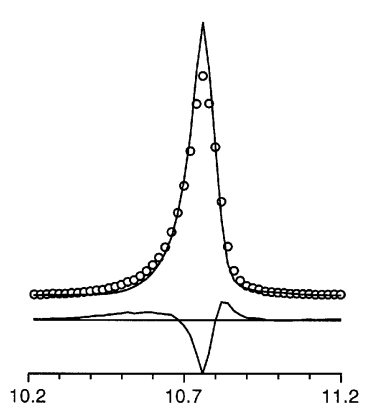

(b)
Fig. 3. The observed (circles), calculated (line) and difference (bottom) profiles for a peak calculated with $(a)$ too large an FWHM and $(b)$ too small an FWHM. The characteristic difference profiles for an FWHM mismatch have '-/+/-' or ' $+/-/+$ ' character. neutron diffractometers have much lower resolution than do constant-wavelength X-ray instruments. A plot of $\mathrm{FWHM}^{2}$ versus $(d, \theta \tan \theta$, etc.) is more parabolic and the line shapes are more Gaussian because of the dominant instrument contribution. In contrast to the $\mathrm{X}$ ray case, $U, V$ and $W$ are easily determined by Rietveld refinement.

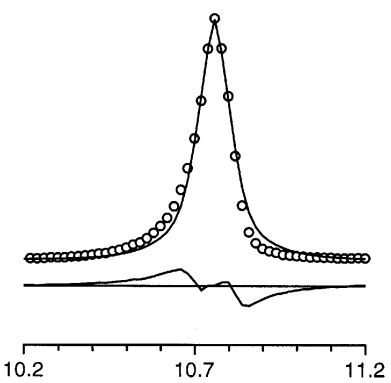

Fig. 4. The observed (circles), calculated (line) and difference (bottom) profiles for a peak calculated with too symmetric a peak-shape function. The characteristic difference profile has a ' + /-' character and is most pronounced for the 'tails' of the peak.

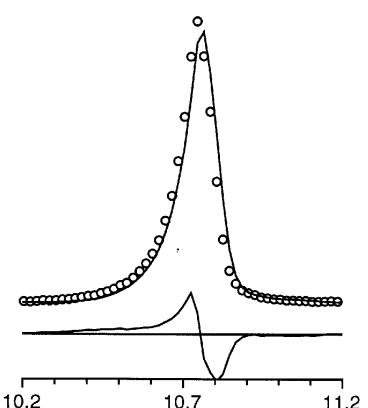

(a)

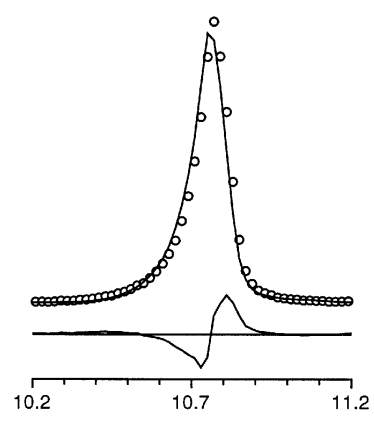

(b)
Fig. 5. The observed (circles), calculated (line) and difference (bottom) profiles for a peak calculated with $2 \theta(a)$ too large and $(b)$ too small. The characteristic difference profiles for a $2 \theta$ mismatch have a ' $+1-$, or a ' $-/+$ ' character.

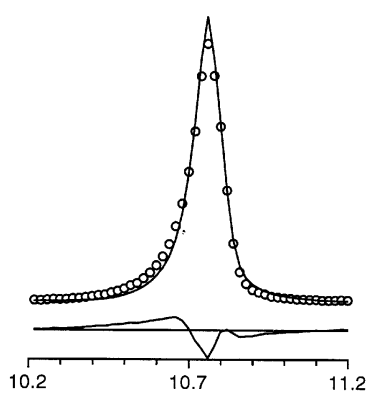

(a)

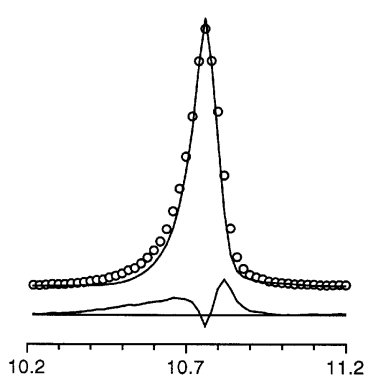

(b)
Fig. 6. The observed (circles), calculated (line) and difference (bottom) profiles for some combinations of incorrect profile parameters (closer to a real refinement situation): (a) an FWHM that is too small combined with a peak asymmetry that is too small, and $(b)$ an FWHM that is too small combined with an intensity that is too small. 
TOF neutron. The more complex Bragg peak shape for TOF neutron data (Von Dreele et al., 1982) can be described using a number of instrument-dependent coefficients, which can be established by Rietveld refinement with data from standard materials. The minimum sample-position bias in the lattice parameters obtained from Rietveld refinement of neutron TOF data is found in the pattern from the set of detectors at the highest angle. Consequently, the effect can be minimized in a multi-data-set refinement by allowing all 'diffractometer constants', except that of the highest-angle detectors, to vary simultaneously with the lattice parameters.

\section{Completing the structural model}

If the structural model is incomplete, difference Fourier maps can be used to locate the missing atoms. In general, refinement of structural parameters should not be started before all (or almost all) atoms have been found. Otherwise, refinement is likely to lead to a false minimum, because the algorithm will attempt to describe all of the electron density with too few atomic positions.

With a partial structural model, the distribution of the intensities of reflections overlapping in the powder pattern can be estimated by assuming that the distribution is the same as that calculated for the partial model. Such a partitioning of the overlapping reflections is easily done in a Rietveld program, because the same procedure is used to calculate the $R_{F}$ values (see below). The more complete the model, the more valid is this approximation. In this way, a pseudo single-crystal dataset (a list of the $h k l$ 's and their corresponding intensities) can be produced and an approximate electrondensity map (albeit model biased) generated via a Fourier transform (using phases calculated from the partial model).

In general, maps calculated from powder data are more diffuse than those calculated from single-crystal data, but they are still quite usable. It should be borne in mind that they are doubly biased towards the structural model, because both the phases (as in the single-crystal case) and the intensity partitioning are taken from the model. By subtracting the electron density calculated for the model from that calculated using the 'observed' reflection intensities, a difference Fourier map (difference electron-density map), which highlights the electron density not accounted for in the model, is produced. Truncation errors are less pronounced in a difference map than in an electron-density map (generated using just the $F_{\text {obs }}$ ), because the missing $\Delta F$ 's are approximately zero, whereas the missing $F_{\text {obs }}$ are not. It is worth mentioning that maps obtained from a maximum entropy reconstruction (see, for example, Kumazawa et al., 1993) are usually less noisy than Fourier maps and may show the missing features more clearly.
The scaling of the calculated pattern to the observed one must be correct in order to obtain a reasonable difference map. To check this, plots of the observed and calculated patterns should always be examined by eye. Automatic scaling will sometimes lead to erroneous results. For zeolites, for example, the low-angle reflection intensities are particularly sensitive to the presence or absence of nonframework species, whereas the highangle reflection intensities are determined primarily by the atoms of the framework. Thus, if the partial model consists of only the atoms of the framework, the highangle reflections are best suited for the determination of the scale factor, even though the resulting fit at low angles looks disastrous. The intensity differences at low angles are real and reflect the incompleteness of the model. These differences can then be used to calculate a difference Fourier map to locate the missing atoms. The scale factor is held fixed (perhaps with minor adjustments as the model becomes more complete) until all missing atomic positions have been added to the model.

\section{Refining the structure}

With a complete structural model and good starting values for the background contribution, the unit-cell parameters and the profile parameters, the Rietveld refinement of structural parameters can begin. Because the global minimum of the least-squares residual function is much shallower with powder data than it is with single-crystal data, and false minima are more prevalent, the refinement needs constant monitoring. A refinement of a structure of medium complexity can require a hundred cycles, while a structure of high complexity may easily require several hundred. Refinement is usually done in sets of two to five cycles at a time. To monitor the progress of a refinement, the two most useful pieces of information are the profile fit and the nature of the parameters shifts (i.e. shift/e.s.d.; are the shifts oscillating, diverging or converging?). The profile fit is best seen in a plot of the observed and calculated patterns, but can also be followed numerically with a reliability factor or $R$ value (see below). It should be emphasized that profile plots are much more informative than $R$ values for guiding a refinement. The difference plots indicate whether a high $R$ value is due to a profileparameter problem (i.e. total intensity is approximately correct but there are differences in the peak form; see Figs. 1-6) or to a deficiency in the structural model (i.e. integrated intensities do not match).

It is difficult to cover all the details of a full refinement, but an approximate strategy can be described. Changes in positional parameters cause changes in structure-factor magnitudes and therefore in relative peak intensities, whereas atomic displacement (thermal) parameters have the effect of emphasizing the highangle region (smaller thermal parameters) or deemphasizing it (larger thermal parameters). It is usually 
advisable to start the refinement of structural parameters with the positions of the heavier atoms and then to try those of the lighter atoms. If the latter refinement converges, all atomic positions in the model can then be refined simultaneously. At this point, the refinement of the somewhat trickier parameters can be attempted.

The scale, the occupancy parameters and the thermal parameters are highly correlated with one another, and are more sensitive to the background correction than are the positional parameters. Refinement of thermal parameters (heavier atoms first) can be attempted, but this can be difficult with X-ray powder data, especially if the $2 \theta$ range is small. Because there is no $\sin \theta / \lambda$ dependence of the scattering cross sections with neutrons, thermal-parameter refinement with neutron data is usually more reliable and even anisotropic refinement is sometimes possible. With X-rays, it is perhaps prudent to constrain the thermal parameters for similar atoms to be equal (at least in the early stages) and thereby reduce the number of thermal parameters required. Occupancy parameters are correspondingly difficult to refine and chemical constraints (e.g. relative occupancies of atoms in a known fragment) should be applied whenever possible. If these parameters are important for the correct interpretation of the structure, simultaneous refinement of multiple data-sets (e.g. one $\mathrm{X}$-ray and one neutron pattern or two X-ray patterns collected at different wavelengths, which change the 'anomalous' scattering properties of one or more atoms) should be considered. By refining a single structure using two independent data-sets containing complementary information, problems of parameter correlation can sometimes be minimized. However, care must be taken that the conditions for both data collections are as similar as possible (e.g. temperature, atmosphere, same sample, etc.), or the joint refinement will not work. Refinement of the profile parameters with the structural parameters is also recommended.

The structure should be refined to convergence. That is, the maximum shift/e.s.d. in the final cycle of refinement should be no more than 0.10 (see below for a discussion on e.s.d.'s). All parameters (profile and structural) should be refined simultaneously to obtain correct estimated standard deviations.

\section{Restraints}

Because powder diffraction data are a one-dimensional projection of three-dimensional data, they suffer from an inherent loss of information. One way to compensate for this loss, at least in part, is to supplement the diffraction data with information from another source. Geometric information (typical bond distances and angles) gleaned from related structures lends itself to such an approach. The information can be used in a Rietveld refinement in two different ways: to increase the number of observations (second data-set consisting of geometric 'observations') or to reduce the number of parameters (e.g. a rigid body). The former is generally preferred because it is easier to implement. The use of restraints in this way not only increases the number of observations, thereby allowing more parameters to be refined, it also keeps the geometry of the structural model sensible.

The set of geometric 'observations' is simply treated as a second data-set and the quantity minimized in the refinement is

$$
S=S_{y}+c_{w} S_{G}
$$

where $S_{y}$ is the weighted difference between the observed $[y(\mathrm{obs})]$ and calculated $[y($ calc $)]$ diffraction patterns (Rietveld residual),

$$
S_{y}=\sum_{\iota} w_{i}\left[y_{i}(\mathrm{obs})-y_{i}(\mathrm{calc})\right]^{2},
$$

$S_{G}$ is the weighted difference between the prescribed $[G(\mathrm{obs})]$ and calculated $[G($ calc $)]$ geometric restraints,

$$
S_{G}=\sum w[G(\text { obs })-G(\text { calc })]^{2},
$$

and $c_{w}$ is a factor that allows a weighting of the geometric observations 'data-set' with respect to the diffraction data-set. In general, the weighting factor $c_{w}$ is set high at the beginning of a refinement when the structure is incomplete or only approximately correct, because the interatomic distances easily become unreasonably long or short at this stage. It can then be reduced during the course of the refinement as the structural model improves.

Experience has shown that if the geometric assumptions are invalid (e.g. a tetrahedral coordination has been assumed, but an octahedral one is actually present), the refinement will not progress satisfactorily. In this case, the restraints need to be examined and reconsidered. Geometric restraints, if used carefully, can enhance a refinement considerably, allowing otherwise impossibly complex structures to be refined successfully. It is imperative, however, that the final structure model fit both the geometric and the X-ray data satisfactorily.

\section{Number of observations (and number of parameters)}

It is difficult to quantify the amount of structural information in a powder diffraction pattern. The intensity at each step along the profile has been measured and, mathematically, each measurement is an observation in the core least-squares algorithm of a Rietveld refinement program. However, only the integrated intensities of the individual reflections can be considered unique observations for the refinement of structural parameters, and these are determined with varying degrees of precision and accuracy depending upon the counting time, the number of steps across the peak (each step represents an independent measurement of the integrated intensity of that reflection) and the amount of 
reflection overlap involved. While it is easy to see that two reflections with the same $2 \theta$ value result in a single peak, and therefore can only be considered to be a single observation, it is less clear how many observations there are when two reflections have similar but not identical $2 \theta$ values. Yet it is important to have some estimate of the amount of information in the pattern in order to judge how many structural parameters can be refined sensibly. The Rietveld algorithm will allow many more parameters to be refined than the data can actually support (because mathematically the number of observations is the number of steps in the profile), so the user has to intervene with common sense. If too many structural parameters have been refined, the fact should be reflected in very large standard deviations (see below). Altomare et al. (1995) have devised a method for estimating the effective number of observations based on the percentage of the area of a reflection that does not overlap with another reflection. While this approach may not have a rigorous basis, it does give the user a reasonable estimate of the number of refinable parameters that the data will support. The observation/ parameter ratio should be at least three and preferably five.

\section{Estimated standard deviations}

It is important to know that the various Rietveld refinement programs calculate e.s.d.'s differently. That is, given the same data and the same structural model, one program will not necessarily produce the same e.s.d.'s for the structural parameters as another. This situation is a result of different interpretations of how the errors are best estimated.

From a purely statistical point of view, each measurement is an independent observation and intensities measured at different points on the same peak are simply two independent measurements of the intensity of that peak. This is then directly comparable to a singlecrystal data-set in which symmetry-equivalent reflections are present or in which the same reflection has been measured more than once. It is important to emphasize that the e.s.d.'s calculated assume that counting statistics are the only source of error. Systematic errors (e.g. bias introduced by some undescribed physics in the experiment or an inadequate background, peak-shape or structural model), which are in fact significant, cannot be estimated. The e.s.d.'s reflect the precision of the refined parameters and not their accuracy. Several of the methods used to calculate the e.s.d.'s deviate from this strict statistical logic in an attempt to allow for systematic errors in the structural model.

A powder diffractionist needs to know which method is used to calculate the e.s.d.'s in the Rietveld program he/she is using and to be aware that opinions vary regarding the most appropriate method; for most purposes that is sufficient. In any publication, the method used to calculate the e.s.d.'s should be stated. It is also important to know how the e.s.d.'s of derived parameters (e.g. interatomic distances and angles calculated from the atomic coordinates) are calculated. The whole correlation matrix, not just the diagonal elements, should be included in the calculation. In cases where the e.s.d.'s are critical to the correct interpretation of small differences (e.g. oxidation states, occupancy parameters or unusual bond lengths that might indicate unusual chemistry), the reader is referred to the papers by Prince (1981, 1993), Scott (1983), Hill \& Flack (1987), Hill \& Madsen (1987), Antoniadis et al. (1990), Bérar (1992) and Cox \& Papoular (1996).

\section{1. $R$ values}

Although a difference profile plot is probably the best way of following and guiding a Rietveld refinement, the fit of the calculated pattern to the observed data can also be given numerically. This is usually done in terms of agreement indices or $R$ values. The weighted-profile $R$ value, $R_{w p}$, is defined as

$$
R_{w p}=\left\{\sum_{i} w_{i}\left[y_{i}(\mathrm{obs})-y_{i}(\mathrm{calc})\right]^{2} / \sum_{i} w_{i}\left[y_{i}(\mathrm{obs})\right]^{2}\right\}^{1 / 2}
$$

where $y_{i}(\mathrm{obs})$ is the observed intensity at step $i, y_{i}$ (calc) the calculated intensity, and $w_{i}$ the weight. The expression in the numerator is the value that is minimized during a Rietveld refinement. If the background has been subtracted, $y_{i}(\mathrm{obs})$ is the net intensity after subtraction, but if the background is refined, $y_{i}$ (obs) [and $y_{i}($ calc $)$ ] is likely to include the background contribution. In the latter case, a high background will automatically produce a low $R_{w p}$ value, because a significant part of the intensity is accounted for by the background function. Thus, the comparison of profile $R$ values from different kinds of powder diffraction experiments can be extremely misleading. For example, $R_{w p}$ for neutron TOF data are often quite small (e.g. a few $\%$ ), while those for laboratory X-ray data are larger (e.g. $\sim 10 \%$ ). This is primarily due to the level of the background. In order to evaluate how well the peaks (which contain the structure-sensitive information) are fitted, an $R_{w p}$ with the background contribution eliminated should also be calculated. Most programs include this feature. In any publication, the type of agreement index used must be clearly specified.

Ideally, the final $R_{w p}$ should approach the statistically expected $R$ value, $R_{\text {exp }}$,

$$
R_{\exp }=\left[(N-P) / \sum_{i}^{N} w_{i} y_{i}(\mathrm{obs})^{2}\right]^{1 / 2}
$$


where $N$ is the number of observations and $P$ the number of parameters. $R_{\exp }$ reflects the quality of the data (i.e. the counting statistics). Thus, the ratio between the two (goodness-of-fit),

$$
\chi^{2}=R_{w p} / R_{\exp }
$$

which is also quoted quite often in the literature, should approach 1. If the data have been 'over-collected' (i.e. errors are no longer dominated by counting statistics), $R_{\exp }$ will be very small and $\chi^{2}$ for a fully refined structure much larger than 1. Conversely, if the data have been 'under-collected' (i.e. collected too quickly), $R_{\exp }$ will be large and $\chi^{2}$ could be less than 1 . Strange $\chi^{2}$ values can also arise from data for which the e.s.d.'s of the counts have been incorrectly calculated (e.g. counts given as counts per second are assumed to be the absolute counts). The final $R_{w p}$ obtained in a structure-free refinement (e.g. using the Le Bail algorithm) is a good indication of the best profile fit of the data that can be obtained, and the $R_{w p}$ in the Rietveld (structural) refinement should approach it.

An $R$ value similar to that reported for single-crystal refinements, based on the agreement between the 'observed' and calculated structure factors, $F_{h k l}$, can also be calculated by distributing the intensities of the overlapping reflections according to the structural model

$$
R_{F}=\sum_{h k l}\left|F_{h k l}(\mathrm{obs})-F_{h k l}(\mathrm{calc})\right| / \sum_{h k l}\left|F_{h k l}(\mathrm{obs})\right| .
$$

This is, of course, biased towards the structural model, but it gives an indication of the reliability of the structure. This quantity is not used actively in the refinement, but should decrease as the structural model improves during the course of the refinement. Similarly, the Bragg-intensity $R$ value

$$
R_{B}=\sum_{h k l}\left|I_{h k l}(\mathrm{obs})-I_{h k l}(\mathrm{calc})\right| / \sum_{h k l}\left|I_{h k l}(\mathrm{obs})\right| .
$$

where $I_{h k l}=m F_{h k l}^{2}(m=$ multiplicity $)$, or its weighted equivalent (Cox \& Papoular, 1996) can be used to monitor the improvement in the structural model.

$R$ values are useful indicators for the evaluation of a refinement, especially in the case of small improvements to the model, but they should not be overinterpreted. The most important criteria for judging the quality of a Rietveld refinement are (i) the fit of the calculated pattern to the observed data and (ii) the chemical sense of the structural model. The former can be evaluated on the basis of the final profile plot (using the complete range of data collected) and the latter on a careful examination of the final atomic parameters. Any publication reporting the results of a Rietveld refinement should always include a plot of the observed intensities, the calculated profile and the difference curve. For Xray data, the intensity scale for the high-angle range, where the observed intensities are very low, should be magnified. Interatomic distances (both bonding and nonbonding) should be reasonable, bond angles sensible and population parameters consistent with the chemical composition of the material. Furthermore, the structure should be consistent with the results of other characterization techniques such as infrared, Raman, ultraviolet, NMR, EPR (electron paramagnetic resonance) and/or mass spectroscopy, thermogravimetric and/ or chemical analysis, electron microscopy, optical or magnetic measurements, etc. Any unusual features in the structural model should be probed using such independent techniques.

\section{Some common problems and where to look for the solution}

Each structure refinement has its own idiosyncrasies and will present problems that require imaginative solutions. However, some problems are of a more general nature and arise in many cases. Probably the most frequent source of difficulty in a Rietveld refinement is an inadvertent error in the input file for the refinement program. If the input file appears to be correct (i.e. the program is in fact doing what you think you told it to do) and the data themselves have been examined critically for possible errors, then perhaps the suggestions below for tackling specific problems will be of some assistance.

\subsection{The background is not well fitted}

Try a different function, background subtraction, or a combination of the two.

\subsection{The peak shapes are poorly described}

(i) Check the difference plot to see if one of the characteristic difference profiles shown in Figs. 2-6 occurs systematically, indicating that a specific profile parameter should be reset or further refined. (ii) Try a different peak-shape function. (iii) Check that there is an asymmetry correction in the peak-shape function. (iv) Check to see if the peak widths are $h k l$ dependent and require a more sophisticated function to describe the $2 \theta$ dependence. A plot of FWHM values of resolved reflections as a function of $2 \theta$ will generally indicate whether or not structural imperfections are causing anisotropic line broadening (e.g. anisotropic size and/or strain, stacking faults) and which functions are best used to describe the angular dependence (see Delhez et al., 1993).

\subsection{There is a mismatch between the peak positions in} the calculated and observed patterns

(i) Determine the unit-cell parameters with an independent measurement using an internal standard. (ii) Check that the $2 \theta$ correction (zero offset and sample displacement) function used is appropriate for the diffractometer geometry. 
12.4. The tails of the peaks in the calculated pattern seem to be cut off prematurely

Try increasing the peak range used in the calculation.

12.5. The relative intensities of a few reflections are too high but none is too low

Check the sample used for data collection. This may indicate a problem with poor particle statistics (i.e. 'rocks in the dust'). The only solution is to recollect the data after regrinding/sieving the sample (and spinning the sample during data collection).

\subsection{There are small unindexed peaks in the diffraction pattern}

(i) If other preparations of the same material have these peaks with similar relative intensities, they are probably not due to an impurity (though it should be verified that these peaks do not arise from the sample holder because the infinite-thickness condition is not fulfilled or because the sample holder moves during a variable-temperature study). Does one (or more) of the unit-cell axes need to be doubled or tripled? Check the space group: can the peaks be indexed in a subgroup of the selected space group? Are other space groups consistent with the assumed systematic absences? How well established are the systematic absences? Are others possible? (ii) If other preparations of the same material do not have these peaks or have them with different relative intensities, they probably belong to a second phase. Try indexing them as a set; try to identify the phase. If its structure is known, try a two-phase refinement.

\subsection{The refinement does not converge}

(i) Look at the observed and calculated profiles carefully. Is the observed peak shape well defined by the profile parameters? Do the peak positions match? Is the background correction sensible? Is the scale factor correct? (ii) Is the structural model complete? If not, try to locate the missing atoms by generating a difference Fourier map before beginning to refine structural parameters. (iii) Check for oscillations in the parameter shifts and apply damping factors as needed. (iv) Examine the covariance matrix for correlations between parameters. If high correlation is present between two variables, it may not be sensible to refine both. High correlations between atomic coordinates may also indicate that the space group is incorrect. (v) Try refining fewer parameters initially. (vi) Try adding geometric restraints (with higher weight for the initial cycles of refinement). (vii) If geometric restraints are already in use, are they correct? (viii) As an alternative to geometric restraints (which increase the number of observations), try using rigid-body descriptions (which reduce the number of parameters). (ix) Set thermal (atomic displacement) parameters at sensible values and hold them fixed (or constrain similar atoms to have identical displacement parameters). (x) Try a different space group. (xi) Is there something fundamentally wrong with the model? (xii) Do the data support the number of parameters being refined?

\subsection{The final structure is not chemically sensible (impossible interatomic distances or displacement factors)}

(i) Try using restraints to keep interatomic distance sensible and increase the weight of the restraints if necessary. (ii) Delete the offending atoms and try generating a difference Fourier map (or a maximum entropy reconstruction) to relocate them. (iii) Try starting from a sensible geometry and refining the structure more cautiously. (iv) Reconsider the model, the restraints and the space group. (v) Try fixing thermal (atomic displacement) parameters at sensible values (or constrain similar atoms to have identical thermal parameters).

\subsection{Refinement converged, but there is an angle- dependent intensity mismatch and/or unreasonable thermal parameters}

(i) Check the Lorentz-polarization correction. (ii) Should an absorption correction be applied? (iii) Is a surface-roughness correction indicated? (iv) Have the atoms been identified correctly? (v) Check the scattering factors used (especially if they have been input by hand). (vi) Is there preferred orientation in the sample?

\section{Conclusions}

Structure refinement using the whole-pattern or Rietveld method is a powerful technique for extracting structural details from powder diffraction data. With present methods, structures with up to 200 structural parameters can be refined successfully, if care is taken and the data are of sufficiently high quality. These guidelines are designed to provide a concise summary of some of the practical aspects of the technique. Small details play an important role in structure analysis using the Rietveld method and attention to these details, though often tedious, is usually rewarded with success.

We would like to thank Christian Baerlocher, Anton Meden and all the members of the IUCr Commission on Powder Diffraction for their valuable criticisms and suggestions as this paper developed. This work was supported in part by the Swiss National Science Foundation (LBM), the US Department of Energy, Basic Energy Sciences, under contract number W-7405-ENG36 (RBVD), and the US Department of Energy, Divi- 
sion of Materials Sciences, under contract number DEAC02-98CH10886 (DEC).

\section{References}

Altomare, A., Burla, M. C., Cascarano, G., Giacovazzo, C., Guagliardi, A., Moliterni, A. G. G. \& Polidori, G. (1996). J. Appl. Cryst. 29, 341-345.

Altomare, A., Cascarano, G., Giacovazzo, C., Guagliardi, A., Moliterni, A. G. G., Burla, M. C. \& Polidori, G. (1995). J. Appl. Cryst. 28, 738-744.

Antoniadis, A., Berruyer, J. \& Filhol, A. (1990). Acta Cryst. A46, 692-711.

Bérar, J.-F. (1992). Accuracy in Powder Diffraction II, NIST Special Publication 846, edited by E. Prince \& J. K. Stalick, pp. 63-67. Washington: USG Printing Office.

Bish, D. L. \& Post, J. E. (1989). Editors. Modern Powder Diffraction, Reviews in Mineralogy, Vol. 20. Washington: Mineralogical Society of America.

Bish, D. L. \& Reynolds, R. C. (1989). Modern Powder Diffraction, Reviews in Mineralogy, Vol. 20, edited by D. L. Bish \& J. E. Post, pp. 73-99. Washington: Mineralogical Society of America.

Buhrke, V. E., Jenkins, R. \& Smith, D. K. (1998). Editors. $A$ Practical Guide for the Preparation of Specimens for X-ray Fluorescence and $X$-ray Diffraction Analysis. New York: Wiley-VCH.

Caglioti, G., Paoletti, A. \& Ricci, F. P. (1958). Nucl. Instrum. 3, 223-228.

Cox, D. E. \& Papoular, R. J. (1996). Mater. Sci. Forum, 228231, 233-238.

Delhez, R., de Keijser, T. H., Langford, J. I., Louër, D., Mittemeijer, E. J. \& Sonneveld, E. J. (1993). The Rietveld Method, edited by R. A. Young, pp. 132-166. Oxford University Press.

Dollase, W. A. (1986). J. Appl. Cryst. 19, 267-272.

Finger, L. W., Cox, D. E. \& Jephcoat, A. P. (1994). J. Appl. Cryst. 27, 892-900.

Hepp, A. \& Baerlocher, Ch. (1988). Aust. J. Phys. 41, 229-236. Hill, R. J. (1991). Powder Diffr. 6, 74-77.

Hill, R. J. (1992). J. Appl. Cryst. 25, 589-610.

Hill, R. J. \& Cranswick, L. M. D. (1994). J. Appl. Cryst. 27, 802844.

Hill, R. J. \& Flack, H. D. (1987). J. Appl. Cryst. 20, 356-361. Hill, R. J. \& Madsen, I. C. (1987). Powder Diffr. 2, 146-162.

Hodeau, J. L., Bordet, P., Anne, M., Prat, A., Fitch, A. N., Dooryhée, E., Vaughan, G. \& Freund, A. (1998). SPIE Proceedings, Vol. 3448, edited by A. T. Macrander, D. M. Mills, A. K. Freund \& T. Ishikawa. In the press.

International Tables of Crystallography (1995). Vol. C. Dordrecht: Kluwer Academic Publishers.
Järvinen, M. J. (1993). J. Appl. Cryst. 26, 525-531.

Kumazawa, S., Kubota, Y., Takata, M., Sakata, M. \& Ishibashi, Y. (1993). J. Appl. Cryst. 26, 453-457.

Langford, J. I. \& Louër, D. (1996). Rep. Prog. Phys. 59, 131-234.

Le Bail, A. (1998). In Microstructure Analysis by Diffraction, edited by H. Bunge, J. Fiala \& R. L. Snyder. IUCr/Oxford University Press. In preparation.

Le Bail, A., Duroy, H. \& Fourquet, J. L. (1988). Mater. Res. Bull. 23, 447-452.

Louër, D. \& Langford, J. I. (1988). J. Appl. Cryst. 21, 430-437.

Madsen, I. C. \& Hill, R. J. (1994). J. Appl. Cryst. 27, 385-392.

Masciocchi, N., Toraya, H. \& Parrish, W. (1991). Mater. Sci. Forum, 79-82, 245-250.

Pawley, G. S. (1981). J. Appl. Cryst. 14, 357-361.

Peschar, R., Schenk, H. \& Capkova, P. (1995). J. Appl. Cryst. 28, 127-140.

Pitschke, W., Hermann, H. \& Mattern, N. (1993). Powder Diffr. 8, 74-83.

Prince, E. (1981). J. Appl. Cryst. 14, 157-159.

Prince, E. (1993). The Rietveld Method, edited by R. A. Young, pp. 43-54. Oxford University Press.

Rietveld, H. (1969). J. Appl. Cryst. 2, 65-71.

Rodriguez-Carvajal, J. (1996). Reference Guide for the Computer Program FullProf. Laboratoire Léon Brillouin, CEA-CNRS, Saclay, France.

Sabine, T. M., Hunter, B. A., Sabine, W. R. \& Ball, C. J. (1998). J. Appl. Cryst. 31, 47-51.

Scardi, P. (1998). In Microstructure Analysis by Diffraction, edited by H. Bunge, J. Fiala \& R. L. Snyder. IUCr/Oxford University Press. In preparation.

Scott, H. G. (1983). J. Appl. Cryst. 16, 159-163.

Shankland, K., David, W. I. F. \& Sivia, D. S. (1997). J. Mater. Chem. 7, 569-572.

Sivia, D. S. \& David, W. I. F. (1994). Acta Cryst. A50, 703-714.

Stephens, P. W. (1999). J. Appl. Cryst. 32. In the press.

Suortti, P. (1972). J. Appl. Cryst. 5, 325-331.

Thompson, P., Cox, D. E. \& Hastings, J. B. (1987). J. Appl. Cryst. 20, 79-83.

Toraya, H., Hibino, H. \& Ohsumi, K. (1996). J. Synchrotron Rad. 3, 75-83.

Von Dreele, R. B. (1998). J. Appl. Cryst. 30, 517-525.

Von Dreele, R. B., Jorgensen, J. D. \& Windsor, C. G. (1982). J. Appl. Cryst. 15, 581-589.

Wilson, A. J. C. (1963). Mathematical Theory of X-ray Powder Diffractometry. Eindhoven: Philips Technical Library.

Young, R. A. (1993). Editor. The Rietveld Method. Oxford University Press.

Young, R. A., Prince, E. \& Sparks, R. A. (1982). J. Appl. Cryst. 15, 357-359. 